\title{
A search for shock-excited molecular hydrogen knots in Chamaeleon I very low mass YSOs ${ }^{\star}$
}

\author{
M. Gómez ${ }^{1}$, P. Persi ${ }^{2}$, A. R. Marenzi ${ }^{2}$, M. Roth ${ }^{3}$, and M. Tapia ${ }^{4}$ \\ 1 Observatorio Astronómico de Córdoba, Laprida 854, 5000 Córdoba, Argentina \\ e-mail: mercedes@oac.uncor.edu \\ 2 Istituto Astrofisica Spaziale e Fisica Cosmica, CNR, Via del Fosso del Cavaliere, 00133 Roma, Italia \\ e-mail: [persi; marenzi]@arm.iasf.cnr.it \\ 3 Las Campanas Observatory, Carnegie Institution of Washington, Casilla 601, La Serena, Chile \\ e-mail: miguel@lco.cl \\ ${ }^{4}$ Instituto de Astronomía, UNAM, Apartado Postal 877, Ensenada, Baja California, CP 22830, Mexico \\ e-mail: mt@astrosen.unam.mx
}

Received 30 January 2004 / Accepted 3 May 2004

\begin{abstract}
We have obtained narrow-band images of three selected areas of the Chamaeleon I dark cloud which harbor very low mass young stars, centered on the $\mathrm{H}_{2}$ and $\mathrm{Br} \gamma$ lines and neighboring continuum as well as on the broad band $K_{\mathrm{s}}$. One region is located in the northern part of the cloud, roughly coinciding with the densest area. The other two regions are in the southern section of the cloud. Our aim is to search for $\mathrm{H}_{2}$ outflows associated with these objects. In the northern region, we found seven new $\mathrm{H}_{2}$ knots, five of which are aligned in the direction of a previously known ${ }^{12} \mathrm{CO}$ molecular bipolar outflow. Further evidence that the class I low mass stellar object ISO-ChaI 192 is the driving source of the molecular flow is given by the presence of a $960 \mathrm{AU}$ long elongated structure at $2.2 \mu \mathrm{m}$ emanating from this star and oriented parallel to the bipolar structure. Another pair of $\mathrm{H}_{2}$ knots, although lying relatively nearby, is not aligned with the outflow direction. They are located on opposite sides of C1-6, a low mass class II object in the northern part of the Chamaeleon I dark cloud. In contrast, we fail to detect any $\mathrm{H}_{2}$ emission object brighter than our sensitivity limit $\left(\sim 6 \times 10^{-32} \mathrm{~W} / \mathrm{m}^{2} \mathrm{~Hz} \operatorname{arcsec}^{2}\right)$ in the two southern areas of the cloud that also harbor several very low mass stars, including two transition stellar/sub-stellar objects. This negative result is probably not surprising in view of the extremely low accretion rates measured for brown dwarfs $\left(\dot{M} \sim 10^{-12}-10^{-9} M_{\odot} \mathrm{yr}^{-1}\right)$. Deeper $\mathrm{H}_{2}$ observations are required to better constraint the outflow event in sub-stellar objects.
\end{abstract}

Key words. stars: formation - stars: low-mass, brown dwarfs - ISM: jets and outflows -

ISM: individual objects: Chamaeleon I

\section{Introduction}

Outflows (i.e., optical jet/HH objects, $2.12 \mu$ m molecular hydrogen and CO bipolar structures) usually accompany the formation of a recently born low mass $\mathrm{T}$ Tauri star. Furthermore, it is widely accepted that every single star is associated with an outflow event at least during the very early stage of its evolution (cf. Bontemps et al. 1996).

Brown dwarfs (i.e., sub-stellar mass objects) may form in a similar manner as low mass stars surrounded by circumstellar disks (Muzerolle et al. 2000; Natta \& Testi 2001; Muench et al. 2001; Jayawardhana et al. 2003; Liu et al. 2003). The standard star-like formation mode, however, involves the presence of outflows. When a dense core collapses, material with a low specific angular momentum falls

* Based on observations collected at the European Southern Observatory, La Silla, Chile, ESO proposal 71.C-0144 and at Las Campanas Observatory, Chile. into the center while higher angular momentum gas forms a circumstellar disk. A wind emanating from the star-disk system clears material away from the rotational axis and produces optical jets and molecular bipolar outflows (Terebey et al. 1984; Shu et al. 1987, 2000; Königl \& Pudritz 2000). However, so far little evidence of the association of brown dwarfs with outflows has been found (Fernández \& Comerón 2001; Barrado y Navascués et al. 2004). In this contribution, we present a search for $\mathrm{H}_{2}$ molecular outflows associated with very low mass stars (with masses $\lesssim$ few tenths of a solar mass) and objects close to the H-burning limit in the Chamaeleon I dark cloud.

In particular, we investigate three areas of Chamaeleon I, a dark cloud at a distance of $160 \mathrm{pc}$ (Whittet et al. 1997). The first region, located in the northern part, corresponds to the densest zone of the cloud $\left(A_{\mathrm{V}} \sim 17\right.$, Jones et al. 1985). It harbors a group of about eight previously known pre-main sequence stars in addition to about six non-classified 
Table 1. Coordinates of the observed areas.

\begin{tabular}{llll}
\hline \hline Region & $\alpha(2000.0)$ & $\delta(2000.0)$ & Low mass objects and infrared sources \\
\hline Northern area & $11: 09: 29.4$ & $-76: 33: 28$ & $\begin{array}{l}\text { ISO-ChaI 192, Hn 11, HM 23, WW Cha, Hn 10, } \\
\text { ISO-ChaI 225, C1-6, C1-2 }\end{array}$ \\
& & $-77: 22: 40$ & $\begin{array}{l}\text { Ced 110-IRS4, Ced 110-IRS6, ISO-ChaI 97, } \\
\text { Bouthern area A }\end{array}$ \\
$11: 07: 16.5$ & & $-77: 30: 34$ & ISO-ChaI 138/143 \\
\hline Southern area B & $11: 08: 21.1$ & - ISO-ChaI 86 \\
\hline
\end{tabular}

Note: Units of right ascension are hours, minutes, and seconds, and units of declination are degrees, arcminutes, and arcseconds.

near-infrared sources (Persi et al. 1999). Among these objects is ISO-ChaI 192, one of the few class I sources identified so far in the cloud and the likely exciting source of the outflow lying in the region (Persi et al. 1999; Mattila et al. 1989).

The other two areas are in the southern part of the Cha I region. One of them includes at least two objects with masses close to the H-burning limit (ISO-ChaI 138/143; Gómez \& Persi 2002). The third region surrounds Ced 110/IRS 4 and comprises a handful of very low mass stars and near-infrared objects (Persi et al. 2001).

In Sect. 2 we briefly describe the observations and the data reduction process. In Sect. 3 we show and discuss the results, and in Sect. 4 a summary and our conclusions are presented.

\section{Observations}

The three regions of the Chamaeleon I dark cloud were observed on April 17-18, 2003 with the ESO NTT near-infrared spectrograph/imaging camera SOFI (Son OF ISAAC). SOFI uses a Hawaii $\mathrm{HgCdTe} 1024 \times 1024$ array that in the large field covers $\sim 4.94^{\prime} \times 4.94^{\prime}$ with a plate scale of $0.292^{\prime \prime}$ per pixel. We used the $\mathrm{H}_{2}(2.12 \mu \mathrm{m})$ and the adjacent continuum $\left(\mathrm{H}_{\text {cont }}\right)$ at $2.09 \mu \mathrm{m}$ filters. These filters have bandwidths of 0.028 and $0.020 \mu \mathrm{m}$, respectively. We acquired eight $2 \times 30 \mathrm{~s}$. exposures in the narrow band filters. We offset the telescope between consecutive exposures by $30^{\prime \prime}$, restricting these movements within a square box centered on the central field position. Table 1 gives central coordinates for the observed regions as well as the names of the low mass young stellar objects and infraredselected candidate members of the cloud lying in these areas.

Additional images of five selected targets within these areas were obtained between May 15 and 19, 2003 with PANIC (Persson's Auxiliary Nasmyth Infrared Camera) a near-infrared (1-2.5 $\mu \mathrm{m}$ ) imager on the Magellan Clay $6.5 \mathrm{~m}$ telescope at Las Campanas (Chile). PANIC has a Rockwell $1024 \times$ 1024 Hawaii array that provides a $2^{\prime} \times 2^{\prime}$ field of view with $0.125^{\prime \prime}$ per pixel. We used the $K_{\mathrm{s}}, \mathrm{H}_{2}$, and Br $\gamma$ filters with bandwidths of $0.324,0.0235$ and $0.0224 \mu \mathrm{m}$, respectively. We typically obtained 9 dithered frames of $5 \mathrm{~s}$. at the $K_{\mathrm{s}}$ filter and of $120 \mathrm{~s}$. each at the narrow-band filters. We offset the telescope by 6 " between consecutive exposures. We also observed a few standards each night from the list of Persson et al. (1998) to calibrate the data.

We processed the data using standard techniques with the software package IRAF ${ }^{1}$. Each program frame, obtained

\footnotetext{
${ }^{1}$ IRAF is distributed by the National Optical Astronomy Observatory, which is operated by the Association of Universities for
}

with SOFI or with PANIC, was divided by a normalized flatfield frame appropriate for each filter. Then a flattened sky frame was subtracted. The flat-field frames were obtained subtracting identical exposure time images in each filter, taken with the lamps on and off. A set of two on-and-off-lamp frames were taken in each filter and then averaged. Sky frames were generated from a median-filtered set of 8 and 9 (NTT and Clay data, respectively) images in each narrow-band filter. To produce a combined image in each filter from the dithered frames, we aligned them using the IRAF subroutines GEOMAP and GEOTRAN and then added the co-aligned frames together.

\section{Results}

\subsection{The northern area}

Figure 1 shows our $\mathrm{H}_{2}$ and $2.09 \mu$ m continuum images obtained with SOFI, corresponding to the northern region of the cloud. We identified four shocked regions (A, B, C, and D) by comparing the $2.12 \mu \mathrm{m}$ with the continuum frame. In the cases of regions $\mathrm{B}$ and $\mathrm{C}$, we found sub-structures or knots (indicated with the numbers 1,2 and 1,2,3 respectively) that we tentatively associate with the same events. Figure 1 also identifies previously known infrared sources in the region. In Figs. 2-4 we show our $\mathrm{H}_{2}, K_{\mathrm{s}}$, and $\mathrm{Br} \gamma$ images obtained with the Magellan Clay telescope for six of the knots. Our Clay images for the region $\mathrm{D}$ add no further details about this knot. It lies very close to the bright star HM 23. This, in combination with the small dithering offset used for the Clay observations ( $\left.6^{\prime \prime}\right)$, prevented us from obtaining a smoother sky-subtracted image to better enhance this faint object. The knots are visible in the $\mathrm{H}_{2}$ image, barely detected at $K_{\mathrm{s}}$ and do not show up in the $\mathrm{Br} \gamma$ filter.

Table 2 provides coordinates and $\mathrm{H}_{2}$ fluxes in a 2 " radius aperture for theses objects. We derived coordinates relative to the known stars in the region with an estimated error of $\lesssim 1^{\prime \prime}$, except for knot $\mathrm{D}$ for which the listed coordinates have an uncertainty of $\lesssim 1.5^{\prime \prime}$. We assume an uncertainty of about $20 \%$ in our flux calibration. The shocked region $\mathrm{D}$ is marginally detected in our images and thus we are unable to measure its flux. The sensitivity limit at $2.12 \mu \mathrm{m}$ is $\sim 6 \times 10^{-32} \mathrm{~W} / \mathrm{m}^{2} \mathrm{~Hz} \operatorname{arcsec}^{2}$ $(1 \sigma, 1 \mathrm{~s})$ for both the Clay/PANIC and NTT/SOFI data.

Apart from the $2.12 \mu \mathrm{m}$ line, near-infrared shocked emission nebulae usually have several relatively weak $\mathrm{H}_{2}$ lines within the $K_{\mathrm{s}}$ bandwidth (Gredel 1994; Nisini et al. 2002). The line at $2.12 \mu \mathrm{m}$ is the strongest and dominates the flux in $K_{\mathrm{s}}$; thus, these objects appear very faint in this broad-band filter.

Research in Astronomy, Inc. under contract to the National Science Foundation. 

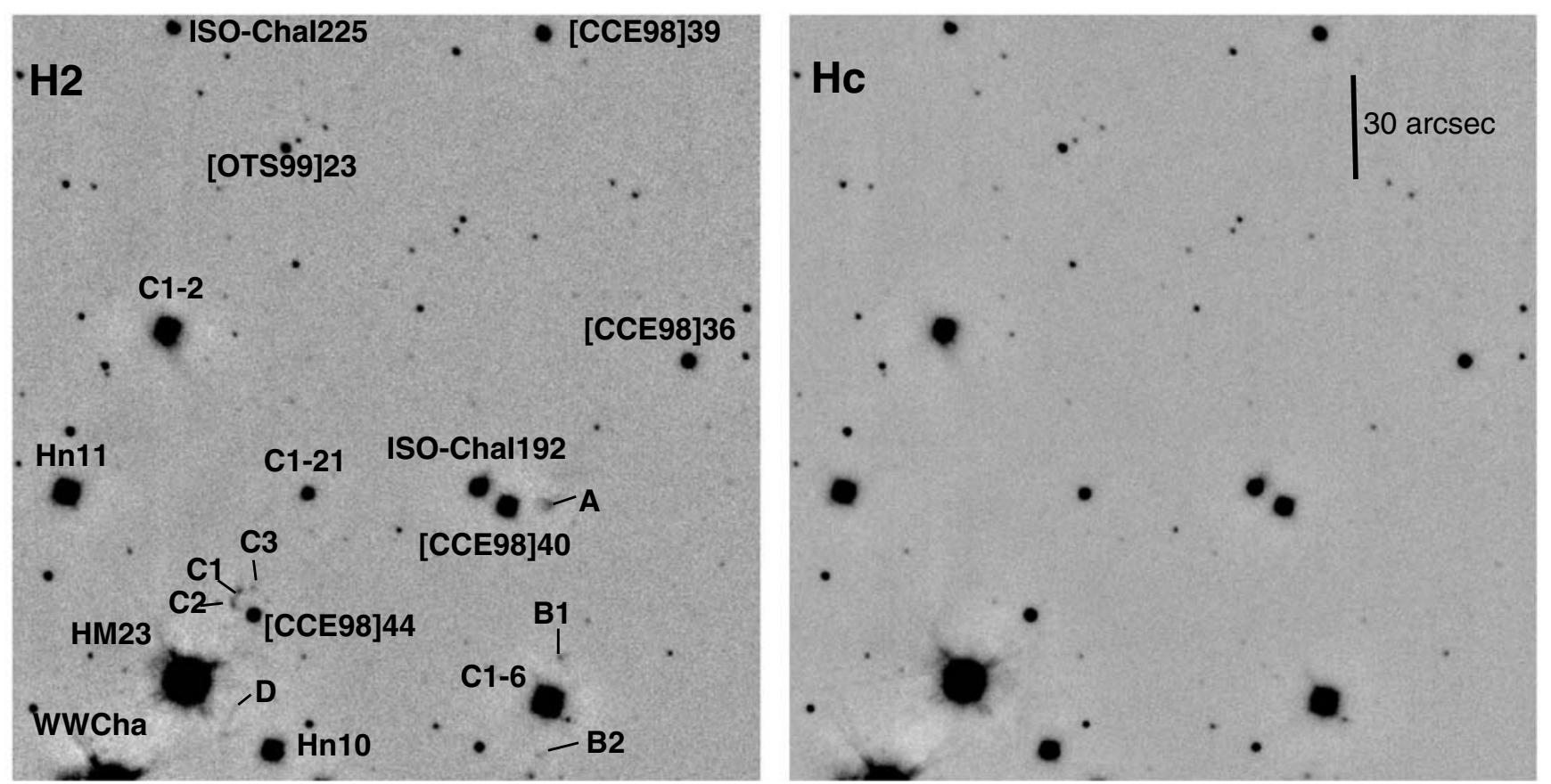

Fig. 1. NTT $\mathrm{H}_{2}$ and $2.09 \mu$ m continuum images of the HM 23/ISO-ChaI 192 region (Northern Area in Table 1), showing a field of 231" $\times 231^{\prime \prime}$. $\mathrm{N}$ is up and $\mathrm{E}$ to the left. Previously known infrared sources and young stellar objects in the region are identified in the $2.12 \mu \mathrm{m}$ image.

Within the bandwidth of the $\mathrm{Br} \gamma$ filter, $\mathrm{H}_{2}$ nebulae often have an emission line at $2.154 \mu \mathrm{m}$, in general a factor of 100 weaker than at $2.12 \mu \mathrm{m}$ (see, for example, Nisini et al. 2002). This line, if present in these new knots, is probably too weak for detection.

Knot A shows an extended morphology. B1 and B2 are two small and diffuse objects located on opposite sides of star C1-6. Knots C1, C2, and C3 seem to delineate an arc-shape structure and knot $\mathrm{D}$ is a diffuse extended object located roughly midway between HM 23 and Hn 10 (see Fig. 1). Although the latter is very faint, it was detected in both the SOFI and the $\mathrm{Clay}_{2}$ images, suggesting that it is a real feature.

Figure 5 shows the $H-K_{\mathrm{s}}$ vs. $J-H$ diagram for the sources identified in Fig. 1 as well as a few other objects (ISOChaI 165, [CCE98] 48, GK-1, ISO-ChaI 193, and Hn 9) located nearby. The photometric data were obtained from the literature (see Carpenter et al. 2002, and references therein). Solid symbols correspond to known young sources and open circles to non-classified infrared sources. All the objects except ISO-ChaI 192 and GK-1 (ISO-ChaI 233), are class II members of the cloud. ISO-ChaI 192 is the only class I object (Persi et al. 1999) while GK-1 may be class II or III (Persi et al. 2000). On this color-color plot we indicate the location of the objects that might be physically associated with the identified knots, judging from their relative location, color excesses, and $\mathrm{H} \alpha$ emission equivalent width or class II spectral energy distribution type. ISO-ChaI 225, Hn 9, and ISO-ChaI 165 are located relatively far away from the detected $\mathrm{H}_{2}$ knots and are not labeled in Fig. 5, though they show near-infrared color excesses.

The identification of the exciting sources for these knots is not easy as many young stars lie in close vicinity. However, at least five of the knots (A, C1, C2, C3, and D) are roughly located in the direction of the large-scale ${ }^{12} \mathrm{CO}(1-0)$ outflow detected by Mattila et al. (1989) as seen in Fig. 6. The other two knots (B1 and B2) also lie relatively nearby but are not aligned in the $\mathrm{CO}$ outflow direction. The nearest star to knot $\mathrm{A}$ is [CCE98] 40, a source without significant near-infrared excess (Carpenter et al. 2002; Gómez \& Mardones 2003). Similarly, the nearest star to knots $\mathrm{C} 1, \mathrm{C} 2$, and C3, namely [CCE98] 44, shows the same photometric characteristics as [CCE98] 40. Low resolution near-infrared spectra of these central objects are featureless with no emission lines (Gómez \& Mardones 2003). However, these stars have been proposed as candidate young members of the cloud by Cambrésy et al. (1998) based on the DENIS IJK magnitudes and colors.

Figure 4 shows a faint point-like source (identified as IRC) in the $K_{\mathrm{s}}$ and $\mathrm{Br} \gamma$ images. Its coordinates are: $\alpha=11^{\mathrm{h}} 09^{\mathrm{m}} 09.3$, $\delta=-76^{\circ} 34^{\prime} 01^{\prime \prime}(\mathrm{J} 2000) ; K_{\mathrm{s}} \sim 17.5$. This object, with apparent $\operatorname{Br} \gamma$ emission on a faint continuum, may be a young brown dwarf of the cloud. Whether this star is responsible for powering knots $\mathrm{C} 1$ and $\mathrm{C} 2$ is uncertain. We expect extremely low mass ejection rates (if any) from this source (see Sect. 3.3) and thus it is unlikely to produce this shocked region. However, this is a possibility that may be worth exploring with additional higher sensitivity and better resolution data. Knot D lies roughly midway between HM 23 and Hn 10, two class II sources of the cloud. Thus, any of these stars may be associated with the $\mathrm{H}_{2}$ knot although this is not the interpretation we favor, as will be discussed further on. Knots B1 and B2 are located on opposite sides of the class II object C1-6 (ISO-ChaI 189), immediately suggesting this star as their driving source. C1-6 has a featureless near-infrared spectrum with both $\mathrm{Pa} \beta$ and $\mathrm{Br} \gamma$ in emission (Gómez \& Mardones 2003). These authors estimated a mass of $0.205 M_{\odot}$ for C1-6.

Persi et al. (1999) argued that the ${ }^{12} \mathrm{CO}$ outflow discovered by Mattila et al. (1989) is powered by the class I star 

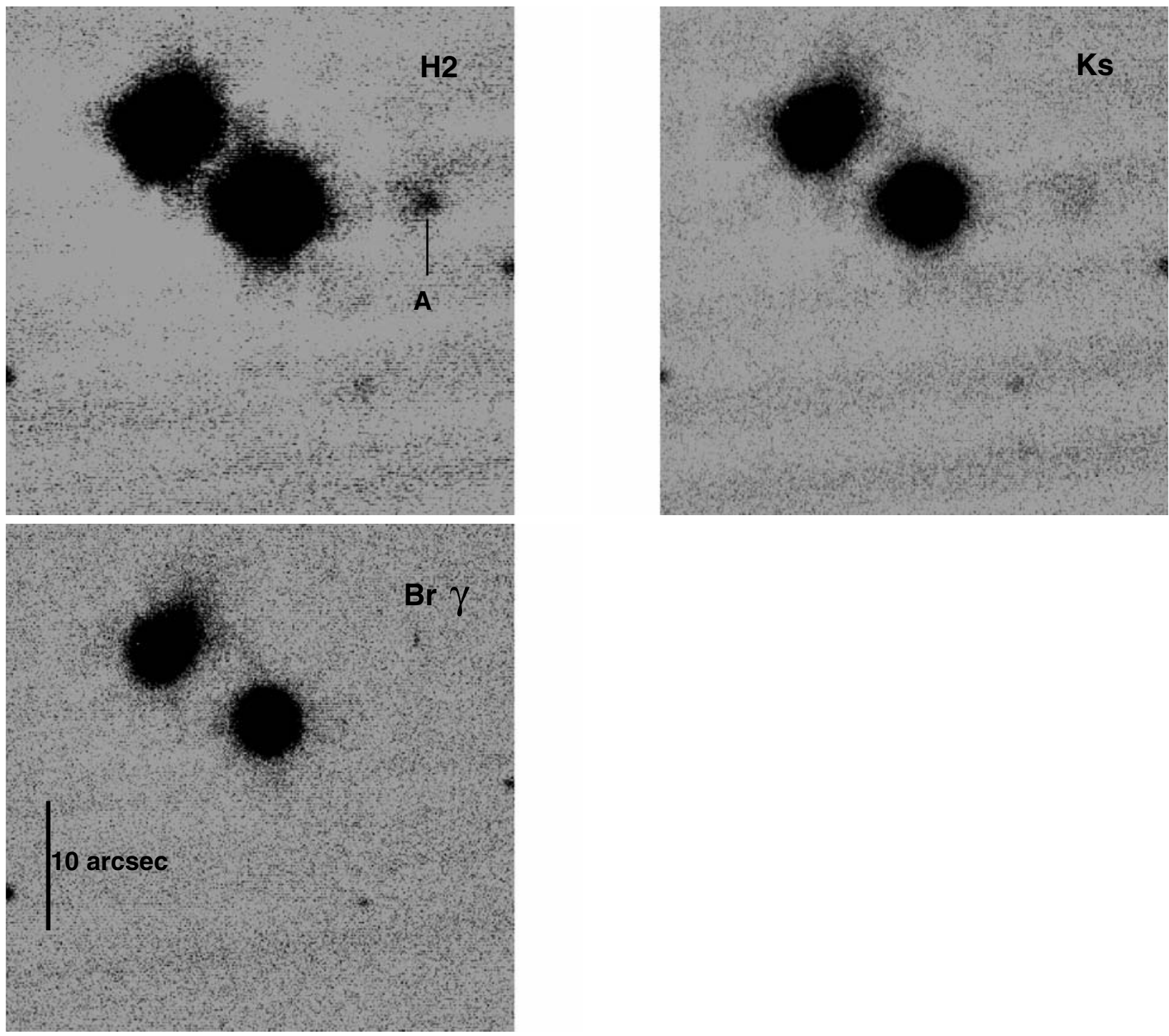

Fig. 2. Magellan (Clay) $\mathrm{H}_{2}, K_{\mathrm{s}}$ and $\mathrm{Br} \gamma$ images of knot A. The field of view is $40^{\prime \prime} \times 40^{\prime \prime} . \mathrm{N}$ is up and $\mathrm{E}$ to the left.

ISO-ChaI 192 based on its spectral energy distribution, its location nearly midway between the peaks of the $\mathrm{CO}$ red and blue lobes and the presence of infrared extended emission superposed on the stellar object. The Magellan Clay images of ISO-ChaI 192 appear elongated towards the northwest, particularly in the $\operatorname{Br} \gamma$ filter (see Fig. 2). The same feature is also apparent in the NTT $\mathrm{H}_{2}$ and $2.09 \mu \mathrm{m}$ images (see Fig. 1). In order to determine more accurately the morphology of this extended emission, we processed our Clay $\mathrm{H}_{2}, \mathrm{Br} \gamma$ and $K_{\mathrm{s}}$ as well as the $2.09 \mu \mathrm{m}$ continuum (NTT) images by subtracting the stellar point-spread function (PSF), taken as that of the nearby star [CCE98] 40 normalized to the same flux, from the observed ISO-ChaI 192 image, leaving only a small residual at the center, except in $K_{\mathrm{s}}$ where the residual was more complex. The resulting "false" coronography images, shown in Fig. 7, clearly show an extended elongated structure around the central object. This elongated feature has a size of $\sim 6^{\prime \prime}$ (960 AU at a distance of $160 \mathrm{pc}$ ) extending towards the northwest. We believe that any extended emission beyond $1.3^{\prime \prime}$ is a reliable detection. No counterpart of this extended emission is seen to the southeast. The position angle of this structure is $145^{\circ}-325^{\circ}$ and it has better contrast, looks more collimated and is probably more extended in $\mathrm{Br} \gamma$ than in the other wavelengths. The position angle of the $\mathrm{CO}$ outflow (Mattila et al. 1989) is $148^{\circ}-328^{\circ}$ (see Fig. 6). The agreement is remarkable and very strongly suggests that the collimated nebular emission arising from ISO-ChaI 192 is associated with the CO outflow and that this star is indeed its engine. Furthermore, Gómez \& Mardones (2003) detected molecular hydrogen emission lines in the nearinfrared spectrum of this object, suggesting the existence of shocked regions in the close vicinity of this star. Although the mass determination for this deeply embedded object is very uncertain (see Sect. 3.3) it is probably a very low mass member of the cloud.

As seen in Fig. 6 the shocked regions $C$ and D are roughly aligned along the CO bipolar outflow, ISO-ChaI 192 and the jet-like emission feature emanating from it while knot $\mathrm{A}$ is close to the putative powering star but in a direction perpendicular to the molecular outflow. From its location, region B seems to be uncorrelated to the above system.

It is natural to think of knots $\mathrm{A}, \mathrm{C} 1, \mathrm{C} 2, \mathrm{C} 3$ and D as relatively distant shocked regions originated by the winds 

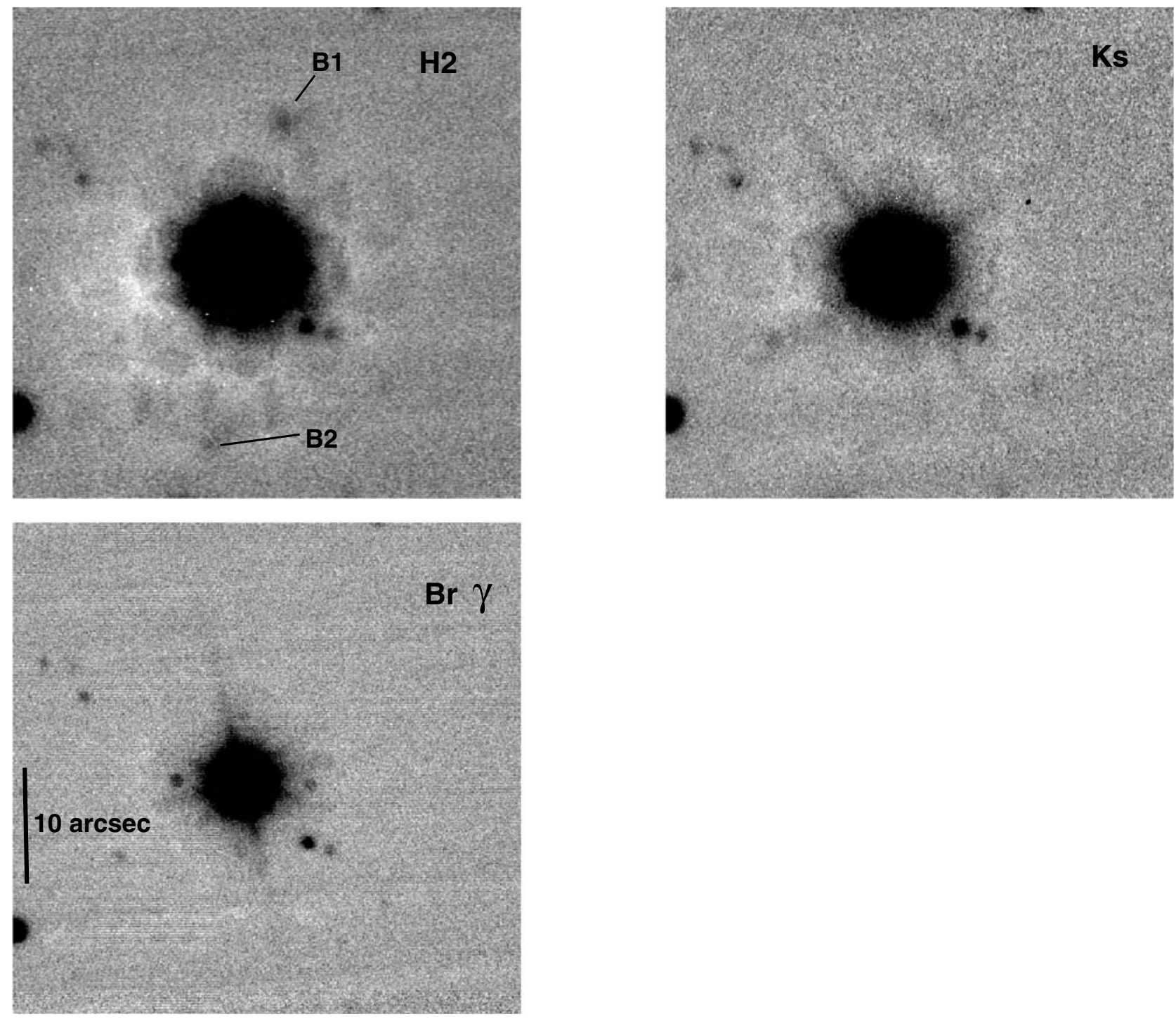

Fig. 3. Magellan (Clay) $\mathrm{H}_{2}, K_{\mathrm{s}}$ and $\mathrm{Br} \gamma$ images of knot B. The field of view is $45^{\prime \prime} \times 45^{\prime \prime}$. $\mathrm{N}$ is up and $\mathrm{E}$ to the left.

emanating from ISO-ChaI 192. Several jet/HH object systems display a chain of knots usually attributed to the repetitive eruptive or FU Orionis episodes of the central star (see, for example, Reipurth \& Bally 2001).

In conclusion, we tentatively associate all the observed $\mathrm{H}_{2}$ knots, except those labeled B1 and B2, with the ${ }^{12} \mathrm{CO}$ outflow of Mattila et al. (1989). This bipolar outflow is powered by the class I low-mass star ISO-ChaI 192 with the reported $\mathrm{H}_{2}$ emission knots originating through shocks along the cavity formed by the flow. On the other hand, C1-6 is probably associated with knots B1 and B2.

\subsection{The southern regions}

Figures 8 and 9 show our SOFI images for the ISOChaI 138/143 and ISO-ChaI 84 (Ced 110 IRS4) regions in the southern part of the cloud (southern areas A and B in Table 1). In addition to ISO-ChaI 84, Fig. 9 includes several other sources lying in the area: B35, ISO-ChaI 97, ISO-ChaI 86, and Ced 110 IRS6. No $\mathrm{H}_{2}$ knots were found in these regions.
Figures 10 and 11 show the Magellan Clay images corresponding to ISO-ChaI 138/143 and ISO-ChaI 84 (Ced 110 IRS4). Figure 11, particularly in the $K_{\mathrm{s}}$ band, clearly shows the bipolar nebulosity produced by the scattered light in the circumstellar dust surrounding the central star. In addition, the disk is seen, in silhouette, as a dark line around this low mass young object (see also Zinnecker et al. 1999; Persi et al. 2001).

ISO-ChaI 143 and 138 have featureless spectra with $\mathrm{Br} \gamma$ in emission. ISO-ChaI 138 also shows $\mathrm{Pa} \beta$ in emission. Both objects display modest infrared-excess (Carpenter et al. 2002; Gómez \& Persi 2002). The masses for these sources are just above the H-burning limit, 0.085 and $0.09 M_{\odot}$ respectively (Gómez \& Persi 2002).

Persi et al. (2001) analyzed the color-color $H-K_{\mathrm{s}}$ vs. $J-H$ diagram of the ISO-ChaI 84 (Ced 110 IRS4) region (see Fig. 9). Ced 110-IRS6, ISO-ChaI 84 (Ced 110-IRS4) and ISOChaI 86 show significant near-infrared excesses whereas B35 and ISO-ChaI 97 show only modest excess emission. Low resolution near-infrared spectra of these sources are featureless. $\mathrm{B} 35$ has $\mathrm{Pa} \beta$ and $\mathrm{Br} \gamma$ in emission while Ced 110-IRS6 shows 

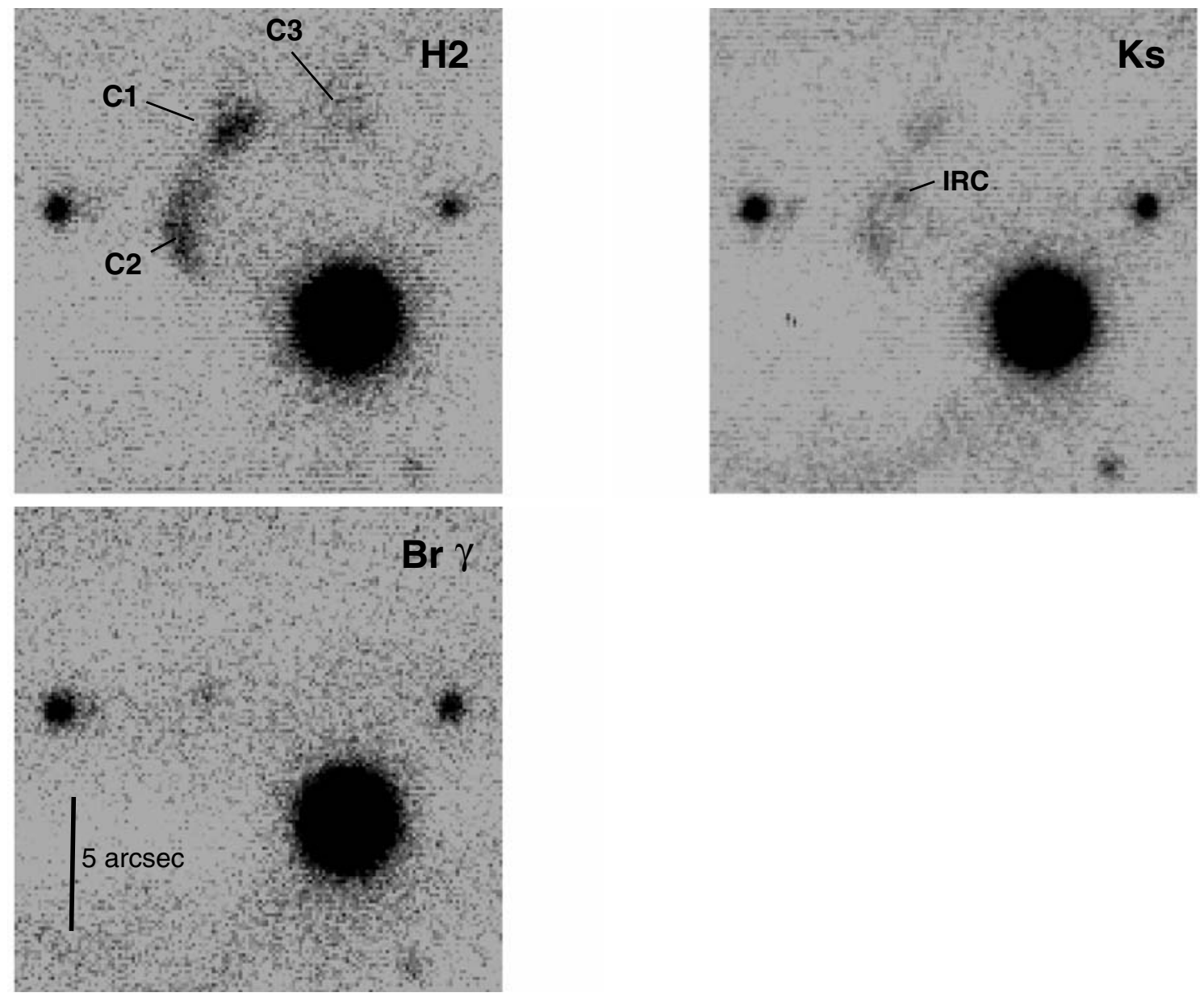

Fig. 4. Magellan (Clay) $\mathrm{H}_{2}, K_{\mathrm{s}}$ and $\mathrm{Br} \gamma$ images of knot $\mathrm{C}$. The field of view is $20^{\prime \prime} \times 20^{\prime \prime} . \mathrm{N}$ is up and $\mathrm{E}$ to the left.

Table 2. Coordinates and fluxes of the $\mathrm{H}_{2}$ knots.

\begin{tabular}{llllcc}
\hline \hline Knot & $\alpha(2000.0)$ & $\delta(2000.0)$ & $m\left(\mathrm{H}_{2}\right)$ & flux $\left[10^{-30} \mathrm{~W} / \mathrm{m}^{2} \mathrm{~Hz}\right]$ & Line flux $\left[10^{-15} \mathrm{~W} / \mathrm{m}^{2}\right]$ \\
\hline A & $11: 09: 22.4$ & $-76: 33: 33$ & 16.80 & 1.32 & 16.8 \\
B1 & $11: 09: 21.6$ & $-76: 34: 19$ & 16.64 & 1.52 & 19.4 \\
B2 & $11: 09: 23.3$ & $-76: 34: 48$ & 17.48 & 0.70 & 8.9 \\
C1 & $11: 09: 48.9$ & $-76: 33: 59$ & 17.04 & 1.05 & 13.4 \\
C2 & $11: 09: 49.4$ & $-76: 34: 03$ & 16.86 & 1.24 & 15.8 \\
C3 & $11: 09: 47.6$ & $-76: 33: 59$ & 18.4 & 0.31 & 4.0 \\
D & $11: 09: 49.6$ & $-76: 34: 38$ & & & \\
\hline
\end{tabular}

Note: Units of right ascension are hours, minutes, and seconds, and units of declination are degrees, arcminutes, and arcseconds.

only the $\mathrm{Br} \gamma$ line in emission. No spectrum is presently available for ISO-ChaI 86. B35 and ISO-ChaI 97 have masses of 0.22 and $0.275 M_{\odot}$, respectively (Gómez \& Mardones 2003).

In summary, although these southern areas of the cloud contain several emission-line very low mass members of the cloud, we found no $\mathrm{H}_{2}$ emission nebulae to our sensitivity limit of $\sim 6 \times 10^{-32} \mathrm{~W} / \mathrm{m}^{2} \mathrm{~Hz} \operatorname{arcsec}^{2}$.

\subsection{Discussion}

For solar-mass $\mathrm{T}$ Tauri stars, mass accretion and outflow events are usually correlated. Edwards et al. (1993) and
Hartigan et al. (1995) note that the intensity of high velocity emissions, such as [OI] $6300 \AA$, thought to arise from an outflow, is correlated with near-IR color excesses usually attributed to dusty circumstellar disks. In addition, Hartigan et al. (1995) found that objects with higher mass accretion rates tend to have higher mass outflow rates (see also Cabrit \& André 1991; Cabrit \& Bertout 1992).

Muzerolle et al. (2000) derived a mass accretion rate of $\sim 5 \times 10^{-12} M_{\odot} \mathrm{yr}^{-1}$ for V410 Anon, a brown dwarf in the Taurus cloud. As these authors note, this mass accretion rate is at least three orders of magnitude lower than that typically corresponding to a $0.5 M_{\odot}$ star (Hartmann et al. 1998). Jayawardhana et al. (2002), White \& Basri (2003) and Muzerolle et al. (2003) have 


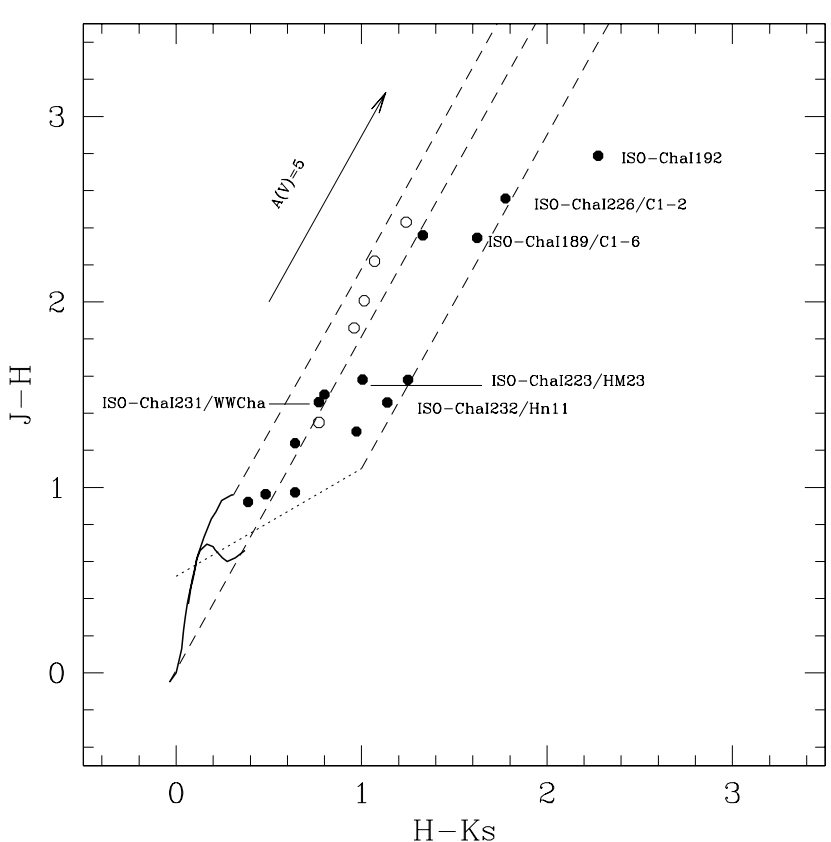

Fig. 5. Color-color $H-K_{\mathrm{s}}$ vs. $J-H$ diagram showing the location of the sources identified in Fig. 1 as well of a few other near-infrared selected objects located relatively nearby.

provided additional examples of accretion disks around substellar objects. In particular, White \& Basri (2003) estimated for their sample upper limit mass accretion rates about 1-3 order of magnitude higher than the value derived for V410 Anon, roughly coinciding with the lower accretion rate $\mathrm{T}$ Tauri stars (Gullbring et al. 1998; White \& Ghez 2001; Hartigan \& Kenyon 2003). From presently available data, they also derived the relation $\dot{M} \propto M$. The mass accretion rate markedly decreases with the mass of the central object (see also Muzerolle et al. 2003). However, the accretion rate for brown dwarfs may decrease more rapidly than for $\mathrm{T}$ Tauri stars (Jayawardhana et al. 2002).

If the accretion/ejection relation holds for sub-stellar objects, one would expect the outflows associated with brown dwarfs to be, on average, less energetic than those associated with low mass pre-main sequence objects. Indeed, Muzerolle et al. (2003) in their sample of very low mass objects (i.e., with mass $0.05-0.2 M_{\odot}$ ) measured emission line equivalent widths (in particular [OI] 6300 and $6363 \AA$ ) similar to those of the least active objects among the more massive $0.5 M_{\odot}$ classical $\mathrm{T}$ Tauri stars (CTTSs). These emission lines may suggest the presence of a wind or outflow associated with the central very low mass source (see also Comerón et al. 2003).

Wolk \& Beck (1990) investigated the possibility of having outflows associated with very low mass stars and substellar objects. They plotted the luminosity of the observed outflows against the luminosity of the driving stars in the sample of Bally \& Lada (1983). They found that these two parameters are correlated although the scatter is relatively large, at least two magnitudes. Extrapolating this correlation into the sub-stellar regime they predicted outflow luminosities of $10^{-5}-10^{-7} L_{\odot}$ for objects with masses below the H-burning limit. According to Shepherd (2003), young low-mass stellar

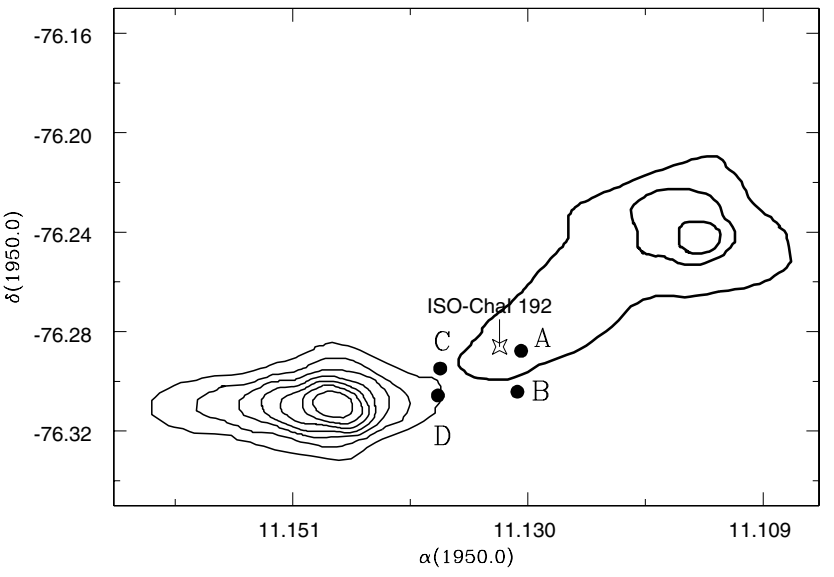

Fig. 6. ${ }^{12} \mathrm{CO}$ wing emission from Mattila et al. (1989) showing also the position of the star ISO-ChaI 192 and of the new shocked excited $\mathrm{H}_{2}$ regions A, B, C and D. Thin contours refer to blueshifted emission and thick contours to redshifted emission.

objects have "typical" outflow mechanical luminosities of $10^{-1}-10^{-3} L_{\odot}$. These central stars have $L_{\text {bol }} \sim$ few $L_{\odot}$ and masses $\gtrsim 0.5 M_{\odot}$. However, Froebrich et al. (2003) found no statistically significant correlation between the source properties and the associated $\mathrm{H}_{2}$ molecular outflow luminosities for a sample of 16 mainly low luminosity (mass) very young (class 0) objects.

At the distance of the Chamaeleon I dark cloud (160 pc), the prediction by Wolk \& Beck (1990) for sub-stellar object outflow luminosities would give fluxes in the range $\sim 10^{-17}$ $10^{-19} \mathrm{~W} / \mathrm{m}^{2}$. Our sensitivity limit $\left(\sim 6 \times 10^{-32} \mathrm{~W} / \mathrm{m}^{2} \mathrm{~Hz} \operatorname{arcsec}^{2}\right)$ is high enough to allow us to detect at least the strongest predicted outflows in the brown dwarf objects in Cha I, provided these fluxes correspond to the $\mathrm{H}_{2}$ flow. Typical $\mathrm{H}_{2}$ knots associated with solar mass $\mathrm{T}$ Tauri stars have integrated fluxes between a few $\times 10^{-14}$ and a few $\times 10^{-18} \mathrm{~W} / \mathrm{m}^{2}$ (Dent et al. 1995; Davis et al. 2000; Eislöffel et al. 2000; Noriega-Crespo et al. 2002). In spite of the large scatter in the $L_{\text {outflow }}$ vs. $L_{\text {bol }}$ relation, for driving sources with masses of about a few tenths of the solar mass we would expect $\mathrm{H}_{2}$ knot fluxes intermediate between those of the brown dwarfs and the solar mass stars.

The present observations revealed seven $\mathrm{H}_{2}$ knots in the northern area of the Cha I cloud with fluxes $\sim 10^{-14} \mathrm{~W} / \mathrm{m}^{2}$, roughly comparable to the brightest small nebulae associated with low mass $\mathrm{T}$ Tauri stars (or central objects with masses $\gtrsim 0.5 M_{\odot}$ ). These fluxes are also higher than the values expected for brown dwarfs based on Wolk \& Beck's (1990) extrapolation. In Sect. 3.1, we suggested ISO-ChaI 192 and C1-6, two low mass members of the cloud, to be the potential driving sources for these knots. The observed $\mathrm{H}_{2}$ flux values may be unexpectedly high for driving sources with masses of about a few tenths of the solar mass as C1-6 and ISO-ChaI 192.

We notice, however, that ISO-ChaI 192 may have some similarities with LS-RCrA 1, a sub-stellar object in the Coronae Australis star-forming complex and with Par-Lup3-4, a $0.12 M_{\odot}$ star, in the Lupus 3 dark cloud. Barrado y Navascués et al. (2004) and Comerón et al. (2003) estimated mass accretion rates of $10^{-10} \lesssim \dot{M} \lesssim 10^{-9} M_{\odot} \mathrm{yr}^{-1}$ for these objects (see 

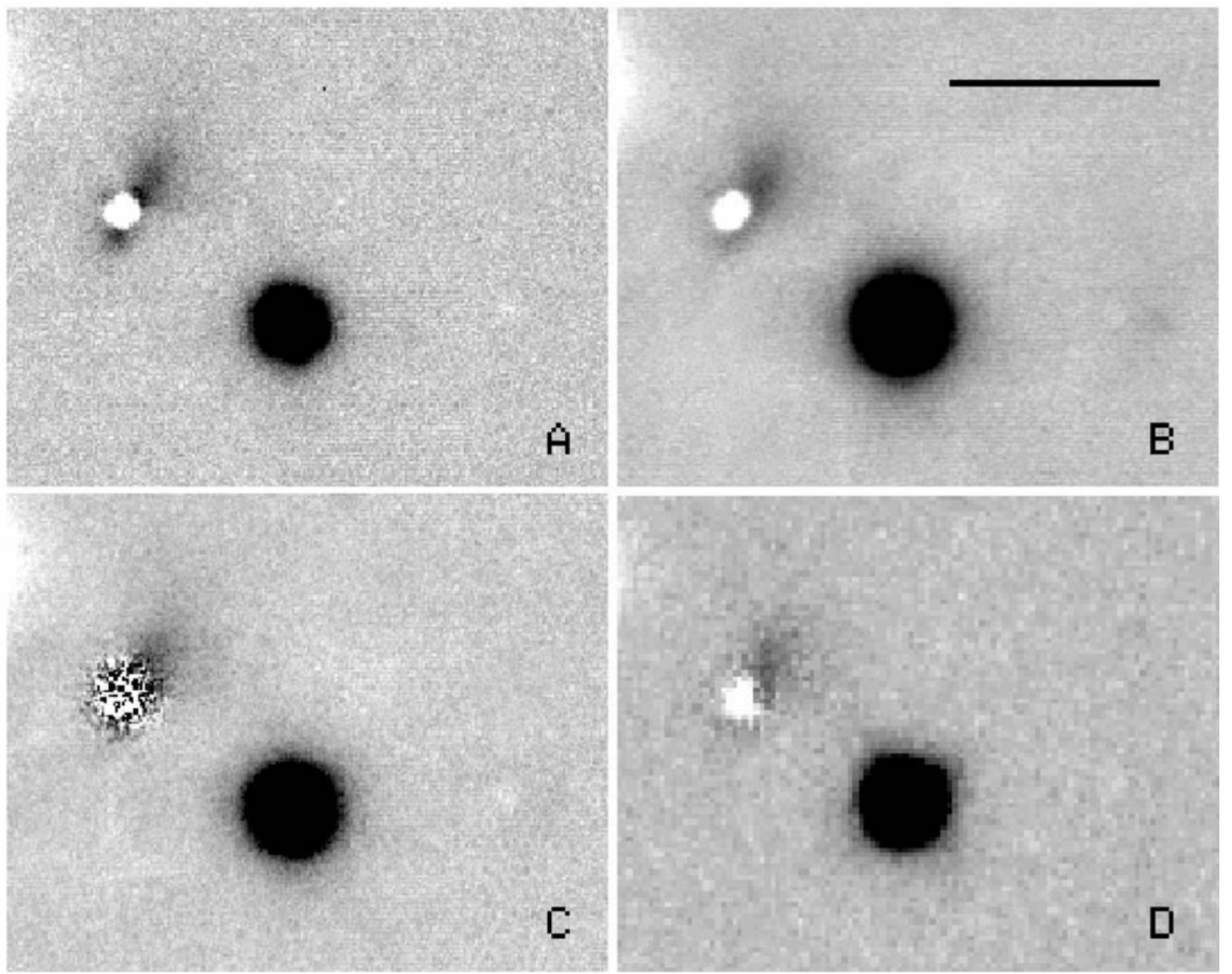

Fig. 7. Composite $\mathrm{Br} \gamma(\mathrm{A}), \mathrm{H}_{2}(\mathrm{~B}), K_{\mathrm{s}}(\mathrm{C})$ and $2.09 \mu \mathrm{m}$ continuum (D) images of the region centered at $\alpha=11^{\mathrm{h}} 09^{\mathrm{m}} 27^{\mathrm{s}} .0, \delta=-76^{\circ} 33^{\prime} 32^{\prime \prime}$ (J2000). The normalized stellar point-spread function has been subtracted from the ISO-ChaI 192 image to show the structure of the elongated emission feature close to it. The bright star is [CCE98] 40. The horizontal bar is 10" long. $\mathrm{N}$ is up and $\mathrm{E}$ to the left.

also Fernández \& Comerón 2001). The spectra of LS-RCrA 1 and Par-Lup3-4 show remarkably strong emission lines, including forbidden lines of [OI], [SII] and [NII], in addition to the $\mathrm{H}_{2}$ line at $2.12 \mu \mathrm{m}$ in the case of LS-RCrA 1 . These emission lines are also detected in solar-mass $\mathrm{T}$ Tauri spectra, although with a much lower intensity (see, for example, Kenyon et al. 1998). They are usually associated with mass loss from stellar winds and shock-excited regions. However, in the cases of LS-RCrA 1 and Par-Lup3-4 the line strengths suggest the presence of outflows with intensities that would be hard to reconcile with such low mass accretion rates. Barrado y Navascués et al. (2004) proposed an edge-on disk surrounding the central star as a means to enhance the outflow signatures over the photospheric features for LS-RCrA 1 (see also Fernández \& Comerón 2001), whereas Comerón et al. (2003) favor a postbirthline evolution substantially modified by accretion in the case of Par-Lup3-4. These objects are also noticeably subluminous and, in consequence, are significantly older than the other young (and in general more massive) members of the clouds which have less conspicuous emission line spectra. A projection factor (i.e., an edge-on disk blocking the light from the central star) or an intrinsic accretion-modified evolutionary process may, at least in principle, account for the unusually faint photospheric continuum in these objects. As remarked by Comerón et al. (2003) the second possibility can explain the observed effect in a qualitative manner but cannot reproduce the observed luminosity. This alternative is suggested by earlier studies of stellar objects with initial masses $>0.15 M_{\odot}$ by Hartmann et al. (1997). In the brown dwarf regime, this may be a much larger and dominant effect that might quantitatively account for the observed difference.

ISO-ChaI 192 shows the molecular hydrogen lines, $\mathrm{H}_{2}$ 1-0 Q(1-8) and $\mathrm{H}_{2}$ 1-0 S(1-0) in emission superimposed on a highly veiled $\left(r_{K}=0-5-0.7\right)$ and practically featureless low resolution $H K$-band spectrum (Gómez \& Mardones 2003). Using the water vapor index, $I_{\mathrm{H}_{2} \mathrm{O}}$ (Comerón et al. 2000), Gómez \& Mardones (2003) derived a mass of 0.19$0.06 M_{\odot}$ for this object. The lower limit was obtained applying the veiling correction of Wilking et al. (1999). Thus the mass determination for this deeply embedded object is very uncertain, although it is probably a very low mass star and not a brown dwarf object of the cloud based on the integrated 


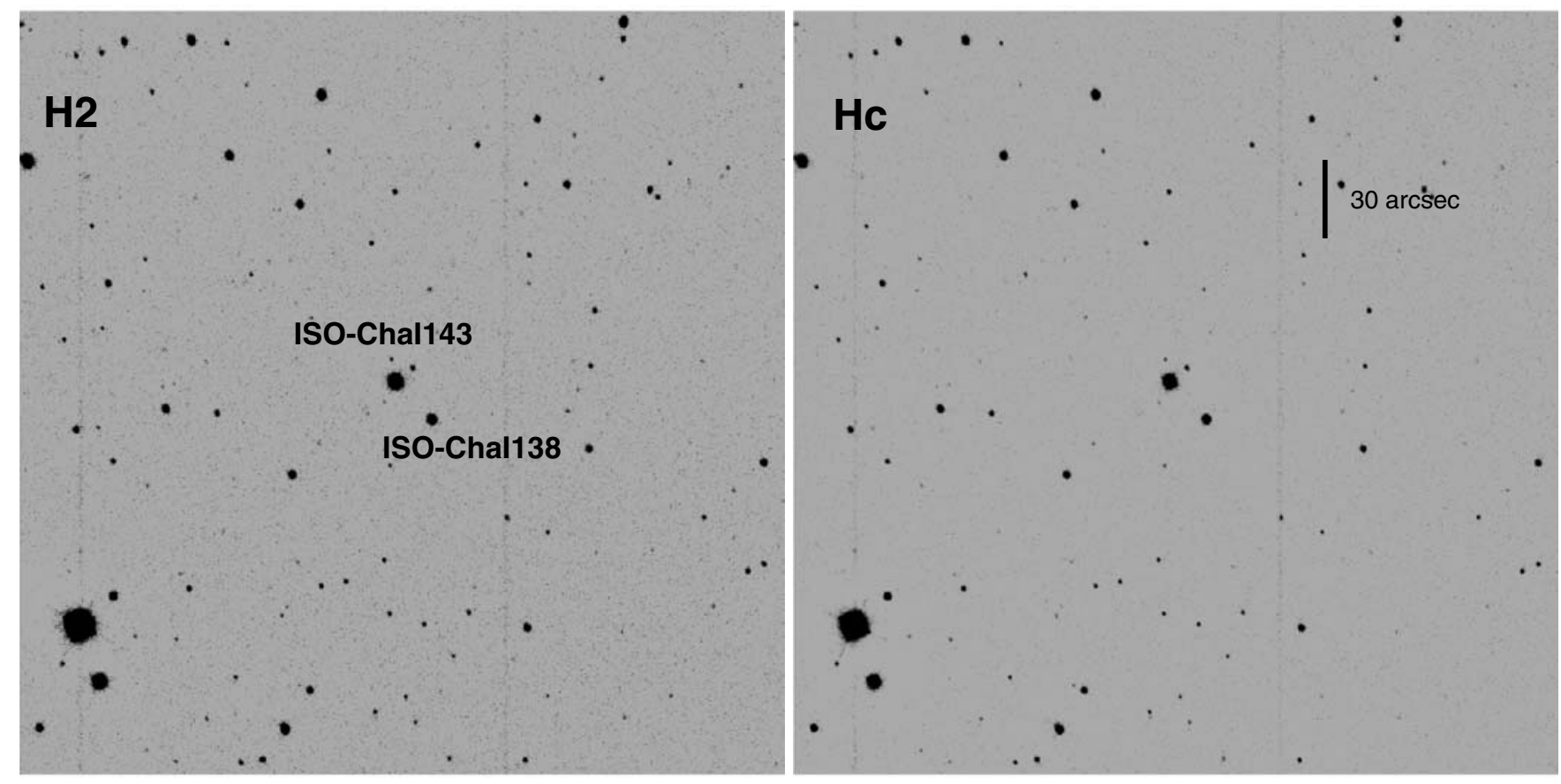

Fig. 8. NTT $\mathrm{H}_{2}$ and $2.09 \mu$ m continuum images of the ISO-ChaI 138/1432 region (Southern Area A in Table 1), showing a field of $231^{\prime \prime} \times 231^{\prime \prime}$. $\mathrm{N}$ is up and $\mathrm{E}$ to the left. The two young stellar objects in the region are identified in the $2.12 \mu \mathrm{m}$ image.
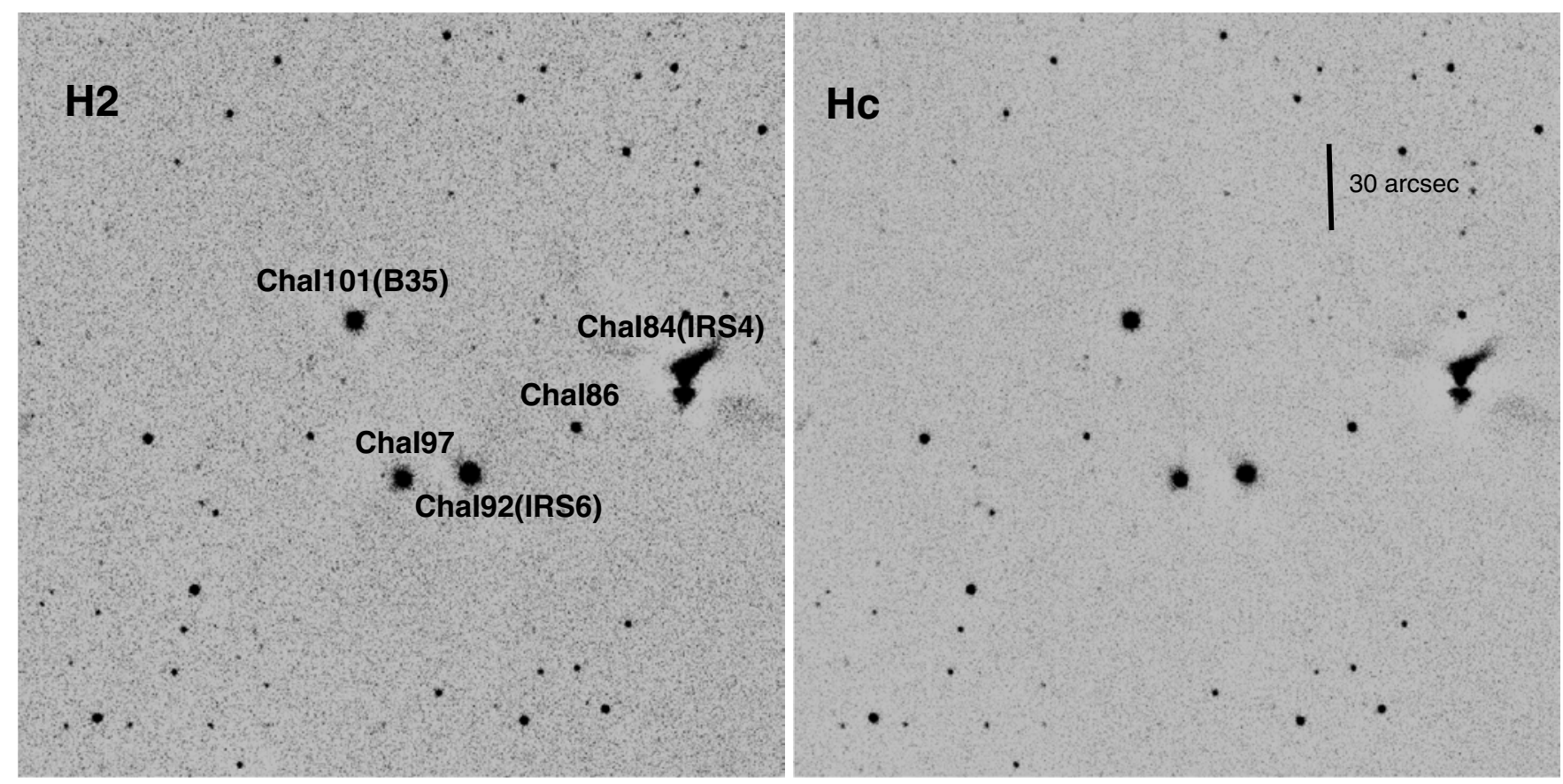

Fig. 9. NTT $\mathrm{H}_{2}$ and $2.09 \mu \mathrm{m}$ continuum images of the ISO-ChaI 84 (Ced 110-IRS4) region (Southern Area B in Table 1), showing a field of $231^{\prime \prime} \times 231^{\prime \prime} . \mathrm{N}$ is up and $\mathrm{E}$ to the left. Previously known infrared sources and young stellar objects in the region are identified in the $2.12 \mu \mathrm{m}$ image.

infrared luminosity from 1 to $18 \mu \mathrm{m}, L \sim 0.6 L_{\odot}$, computed by Persi et al. (1999). However, ISO-ChaI 192 does not seem to be noticeably underluminous or to be much older when compared with the rest of the young cloud members.

In the case of ISO-ChaI 192, a nearly edge-on disk may contribute to tunnel a relatively weak wind towards the polar region and produce a stronger impact zone (brighter $\mathrm{H}_{2}$ knot) in the surrounding parent cloud material. Due to the class I nature of this object (Persi et al. 1999), we may expect a flared disk or an infalling envelope with a bipolar cavity. The shape of the circumstellar material may thus contribute to better constrain (or collimate) the relatively weak wind or outflow emanating from the central source. In addition, an edge-on disk (blocking the star light) or an envelope (scattering the star light in the walls 

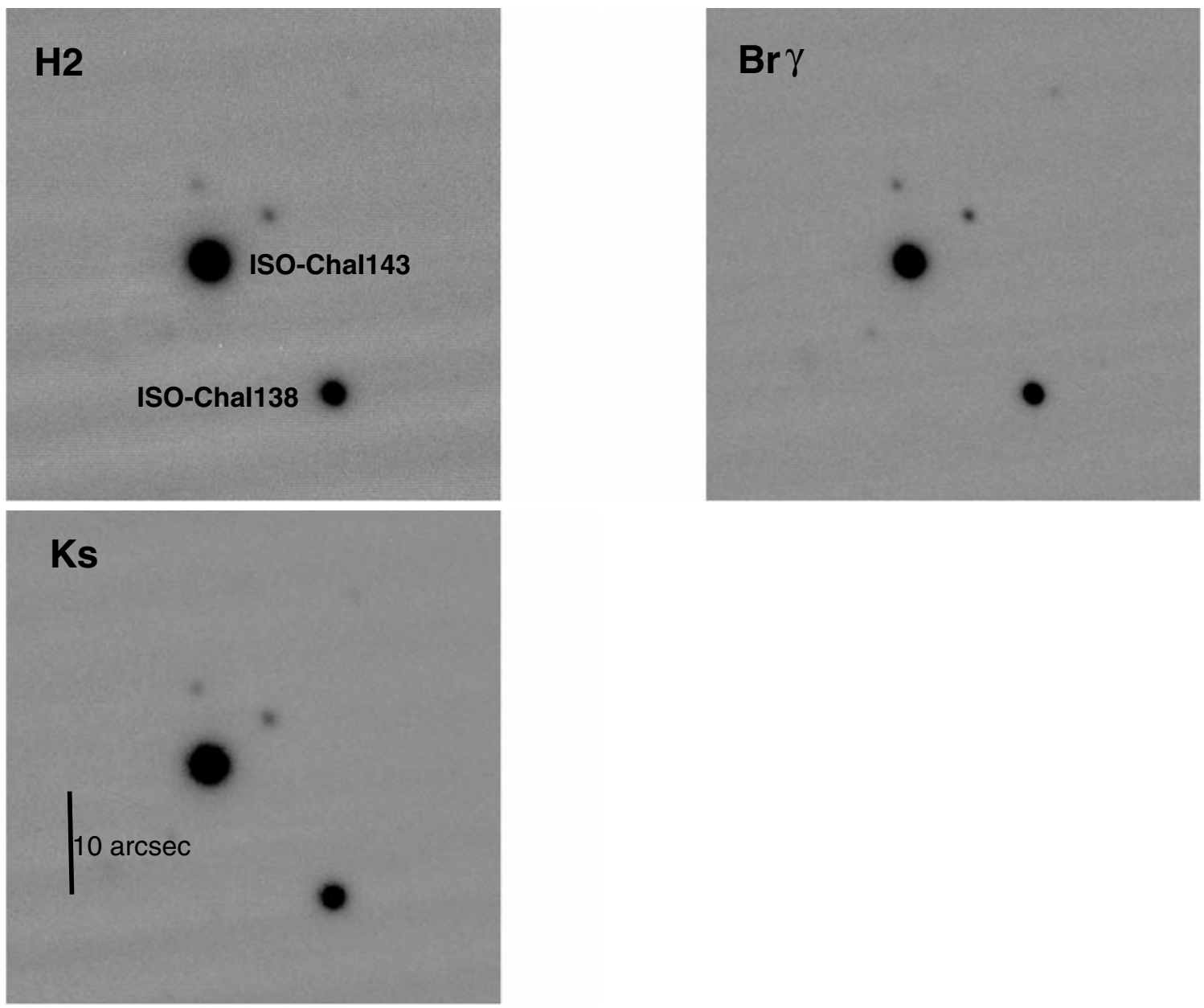

Fig. 10. Magellan (Clay) $\mathrm{H}_{2}, K_{\mathrm{s}}$ and $\mathrm{Br} \gamma$ images of ISO-ChaI $138 / 143$. The field of view is $49^{\prime \prime} \times 49^{\prime \prime}$. $\mathrm{N}$ is up and $\mathrm{E}$ to the left.

of the cavities) would diminish photospheric features relative to the outflow emission lines.

The elongated diffuse structure seen in our point spread function-subtracted image of ISO-ChaI 192 (Fig. 7) has a position angle of $\sim 145-325$ degrees, in agreement with the general direction of the large scale ${ }^{12} \mathrm{CO}$ molecular outflow of Mattila et al. (1989). Although we have no information on the angle of view it is reasonable to argue that the elongation is predominantly along the infalling envelope cavity and that the disk is seen approximately edge-on. A bipolar outflow opens a cavity in the envelope, the light from the central star can thus escape through the cavity and scatter off material producing an elongated structure in the direction of the flow (Whittet et al. 1997). In this scenario, knot A would be located near the equatorial or disk plane and then it might be hard to reconcile with the standard picture in which the outflow follows the rotational axis. However, outflows associated with low mass stars are usually very poorly collimated (Bontemps et al. 1996) and many seem to change the direction of ejection with time (Devine et al. 1997; Gómez et al. 1997; Reipurth \& Bally 2001) sweeping a wider opening angle on the sky. In fact, the outflow of Mattila et al. (1989) is relatively poorly collimated. Knot A lies inside the red lobe of the ${ }^{12} \mathrm{CO}$ molecular outflow and thus it may correspond to an ejection of a wiggling or precessing jet axis (Davis et al. 1994; Arce \& Goodman 2001). Knots C1, C2, C3 and $\mathrm{D}$ would roughly align with the outflow axis in this case, indicating another ejection direction.

Conversely, if the elongation is essentially along the disk, then knot A would be located near the polar region of the stardisk system and the knots $\mathrm{C} 1, \mathrm{C} 2, \mathrm{C} 3$ and $\mathrm{D}$ in the equatorial zone. The scattering models of Whitney \& Hartmann (1992, 1993 ) predict that image elongation should align with the disk for intermediate inclination (i.e., basically no edge-on disks). We favor the first possibility although we caution that higher resolution and deeper sensitivity images are required to better understand the geometry of the system.

The accretion-modified evolution hypothesis does not seem to provide an explanation for the peculiarities observed in ISO-ChaI 192 although we cannot disregard that this effect takes place in this object. As mentioned before, ISO-ChaI 192 is not noticeably subluminous.

The veiling of photospheric absorption features is a direct consequence of accretion in low mass T Tauri stars (see, for example, Hartigan et al. 1995; Gullbring et al. 1998). The high veiling in the ISO-ChaI 192 spectrum $\left(r_{K}=0.5-0.7\right)$ also suggests that the star is probably in an intense accretion phase. This may also intensify the ejection event. We may expect a higher mass accretion rate in ISO-ChaI 192 than in other brown dwarfs (see White \& Ghez 2001; White \& Basri 2003; Muzerolle et al. 2003; Hartigan \& Kenyon 2003) as it is in an earlier 

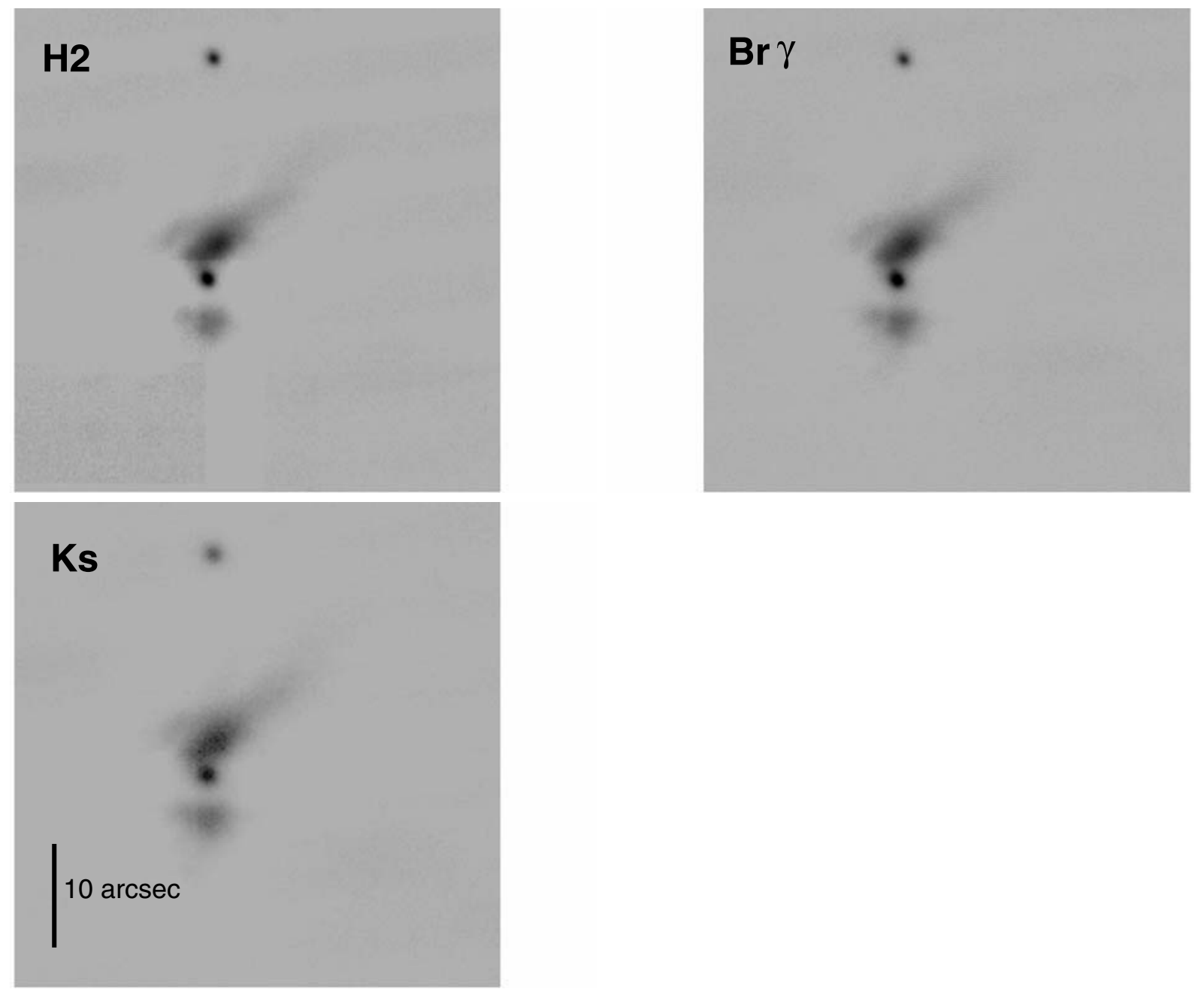

Fig. 11. Magellan (Clay) $\mathrm{H}_{2}, K_{\mathrm{s}}$ and Br $\gamma$ images of ISO-ChaI 184. The field of view is $49^{\prime \prime} \times 49^{\prime \prime}$. $\mathrm{N}$ is up and $\mathrm{E}$ to the left.

stage of the evolution. ISO-ChaI 192 is a class I source (Persi et al. 1999), whereas the great majority of these sub-stellar objects are probably class II sources (see, for example, White $\&$ Basri 2003). For the solar-mass T Tauri stars the mass accretion rate decreases up to 2 orders of magnitude between the class I $\left(\dot{M} \sim 10^{-6} M_{\odot} \mathrm{yr}^{-1}\right.$, Whitney et al. 1997) and the class II $\left(\dot{M} \sim 10^{-8} M_{\odot} \mathrm{yr}^{-1}\right.$, Hartmann et al. 1998) stages of the evolution.

In any event, little is known about the energy associated with outflows from very low mass objects, and although knots A, C1, C2, C3 and maybe D could be unexpectedly bright, their association with ISO-ChaI 192 seems plausible based on the presently available data. Similarly, the fluxes for the knots B1 and B2 might be relatively high for C1-6, a $\sim 0.2 M_{\odot}$ star. However, the prediction by Wolk \& Beck (1990) implies a large scatter of at least two magnitudes, and thus the discrepancy between the expected flux and the actual measurement may not be significant.

Our failure to detect $2.12 \mu \mathrm{m}$ knots in the southern areas of the cloud may be due to our modest sensitivity limit considering the low accretion rate and the weak mechanical luminosities expected for sub-stellar objects. Higher sensitivity $\mathrm{H}_{2}$ images may help to detect molecular outflows associated with the extremely low mass objects in these regions and provide better constraints on the $\mathrm{H}_{2}$ fluxes associated with very low mass stars and young brown dwarf objects.

\section{Summary and conclusions}

We obtained $\mathrm{H}_{2}$ images of three regions in the Chamaeleon I dark cloud containing very low mass members. We found seven $\mathrm{H}_{2}$ knots in the northernmost area observed. Five of these knots are roughly aligned in the direction of the ${ }^{12} \mathrm{CO}(1-0)$ outflow previously detected by Mattila et al. (1989). The other two knots also lie relatively nearby although not in the direction of the $\mathrm{CO}$ flow.

We identify ISO-ChaI 192, the only class I source in the region (Persi et al. 1999), as the exciting star of knots A, C1, $\mathrm{C} 2, \mathrm{C} 3$ and $\mathrm{D}$. This object has also been previously associated to the ${ }^{12} \mathrm{CO}(1-0)$ outflow in the region by Persi et al. (1999). We resolve an elongated structure around ISO-ChaI 192 that perfectly aligns with the $\mathrm{CO}$ outflow direction which provides further support to the suggestion by Persi et al. (1999).

Gómez \& Mardones (2003) also reported the detection of molecular hydrogen emission lines in the $H K$-band spectrum 
of ISO-ChaI 192. Knots A, C1, C2, C3 and D are probably more distant shocked regions associated with the same source.

We notice, however, that the $\mathrm{H}_{2}$ fluxes of these knots are somewhat higher than expected for a "typical" extremely low accretion rate of very low mass object. An edge-on disk may contribute to produce the brightness of this knot and also intensify the outflow signatures (such as the molecular hydrogen lines, $\mathrm{H}_{2}$ 1-0 $\mathrm{Q}(1-8)$ and $\mathrm{H}_{2}$ 1-0 $\left.\mathrm{S}(1-0)\right)$ over photospheric features in the $H K$-band spectrum of the central object. The type of this object (class I) as well as its high veiling $\left(r_{K}=0.5-0.7\right)$ suggest that the central object may be accreting mass at a higher rate than a "typical" class II very low mass or brown dwarf object. Thus mass ejection may be also enhanced.

We propose $\mathrm{C} 1-6, \mathrm{a} \sim 0.2 M_{\odot}$ star in the region, as the exciting source of knots B1 and B2 mainly based on morphological or geometrical arguments. These knots seem to emanate from opposite sides of C1-6.

At the present time and in view of poor understanding of outflows in very low mass stars, the discrepancy between the expected flux and the actual measurement may not be significant. Nevertheless confirmation is required of the physical association of the $\mathrm{H}_{2}$ knots with these central objects. Radial velocities and/or proper motions of the detected $\mathrm{H}_{2}$ knots may help to better understand the kinematics of these objects in relation with the ${ }^{12} \mathrm{CO}(1-0)$ outflow. Further searches and detections of outflows in very low mass and sub-stellar objects will better characterize these events in relation to the ISO-ChaI 192 and $\mathrm{C} 1-6$ cases.

We failed to detect $\mathrm{H}_{2}$ knots associated with several young very low mass stars lying in the two southern areas of the cloud that were observed. These regions harbor ISO-ChaI 138/143, two transition stellar/sub-stellar objects of the cloud and Ced 110 IRS 4 (ISO-ChaI 84) as well as at least two other low mass stars (B35 and ISO-ChaI 97) and near-infrared sources with excess emission (ISO-ChaI 86 and Ced 110-IRS6). This negative result may be due to a modest sensitivity limit $\left(\sim 6 \times 10^{-32} \mathrm{~W} / \mathrm{m}^{2} \mathrm{~Hz} \operatorname{arcsec}^{2}\right)$, in view of the extremely low mass accretion rates derived for brown dwarf objects $\left(\dot{M} \sim 10^{-12}-10^{-9} M_{\odot} \mathrm{yr}^{-1}\right.$, Muzerolle et al. 2000; White $\&$ Basri 2003; Muzerolle et al. 2003). Deeper $2.12 \mu \mathrm{m}$ images of these objects may detect fainter emissions and provide new insight into the outflow events in very low mass and sub-stellar objects.

Acknowledgements. We are grateful to the ESO and Las Campanas staffs for assistance during the observing runs. This work was supported in part by a CNR(Italy)-Conacyt(Mexico) bilateral agreement. M.G. and P.P. also acknowledge financial support form the bilateral project SECyT (Argentina)-MAE (Italy) $21 \mathrm{~F}$ and MT acknowledges UNAM-DGAPA grant IN-105400. This research has made use of the SIMBAD database, operated at CDS, Strasbourg, France.

\section{References}

Arce, H. G., \& Goodman, A. A. 2001, ApJ, 554, 132 Bally, J., \& Lada, C. J. 1983, ApJ, 265, 824
Barrado y Navascués, D., Mohanty, S., \& Jayawardhana, R. 2004, ApJ, 604, 284

Bontemps, S., Andre, P., Terebey, S., \& Cabrit, S. 1996, A\&A, 311, 858

Cabrit, S., \& André, P. 1991, ApJ, 379, L25

Cabrit, S., \& Bertout, C. 1992, A\&A, 261, 274

Cambrésy, L., Copet, E., Epchtein, N., et al. 1998, A\&A, 338, 977

Carpenter, J. M., Hillenbrand, L. A., Skrutskie, M. F., \& Meyer, M. R. 2002, AJ, 124, 1001

Comerón, F., Fernández, M., Baraffe, I., Neuhäuser, R., \& Kaas, A. A. 2003, A\&A, 406, 1001

Comerón, F., Neuhäuser, R., \& Kaas, A. A. 2000, A\&A, 359, 269

Davis, C. J., Mundt, R., \& Eislöffel, J. 1994, ApJ, 437, L55

Davis, C. J., Berndsen, A., Smith, M. D., Chrysostomou, A., \& Hobson, J. 2000, MNRAS, 314, 241

Dent, W. R. F., Matthews, H. E., \& Walther, D. M. 1995, MNRAS, 277, 193

Devine, D., Bally, J., Reipurth, B., \& Heathcote, S. 1997, AJ, 114, 2095

Edwards, S., Strom, S. E., Hartigan, P., et al. 1993, AJ, 106, 372

Eislöffel, J., Smith, M. D., \& Davis, C. J. 2000, A\&A, 359, 1147

Fernández, M., \& Comerón, F. 2001, A\&A, 380, 264

Froebrich, D., Smith, M. D., Hodapp, K.-W., \& Eislöffel, J. 2003, MNRAS, 346, 163

Gómez, M., \& Mardones, D. 2003, AJ, 125, 2155

Gómez, M., \& Persi, P. 2002, A\&A, 389, 494

Gómez, M., Whitney, B. A., \& Kenyon, S. 1997, AJ, 114, 1138

Gredel, R. 1994, A\&A, 292, 580

Gullbring, E., Hartmann, L., Briceño, C., \& Calvet, N. 1998, ApJ, 492, 323

Hartigan, P., Edwards, S., \& Ghandour, L. 1995, ApJ, 452, 736

Hartigan, P., \& Kenyon, S. J. 2003, ApJ, 583, 334

Hartmann, L., Calvet, N., Gullbring, E., \& D'Alessio, P. 1998, ApJ, 495,385

Hartmann, L., Cassen, P., \& Kenyon, S. J. 1997, ApJ, 475, 770

Jayawardhana, R., Mohanty, S., \& Basri, G. 2002, ApJ, 578, L141

Jayawardhana, R., Ardila, D. R., Stelzer, B., \& Haisch Jr, K. E. 2003, ApJ, 126, 1515

Jones, T. L., Hyland, A. R., Harvey, P. M., et al. 1985, AJ, 90, 1191

Kenyon, S. J., Brown, D. I., Tout, C. A., \& Berlind, P. 1998, AJ, 115, 2491

Königl, A., \& Pudritz, R. E. 2000, in Protostars and Planets IV, ed. V. Mannings, A. Boss, \& S. Russell (Tucson: Univ. Arizona Press), 759

Liu, M., Najita, J., \& Tokunaga, A. T. 2003, ApJ, 585, 372

Mattila, K., Liljeström, T., \& Toriseva, M. 1989, in Low Mass Star Formation and Pre-Main Sequence Objects, ed. B. Reipurth (Garching: ESO), 153

Muench, A. A., Alves, J., Lada, C. J., \& Lada, A. E. 2001, ApJ, 558, L51

Muzerolle, J., Briceño, C., Calvet, N., et al. 2000, ApJ, 545, L141

Muzerolle, J., Hillenbrand, L., Calvet, N., et al. 2003, ApJ, 592, 266

Natta, A., \& Testi, L. 2001, A\&A, 376, L22

Nisini, B., Caratti, O., Garatti, A., et al. 2002, A\&A, 393, 1035

Noriega-Crespo, A., Cotera, A., Young, E., \& Chen, H. 2002, ApJ, 580, 959

Persi, P., Marenzi, A. M., Kaas, A. A., et al. 1999, AJ, 117, 439

Persi, P., Marenzi, A. M., Olofsson, G., et al. 2000, A\&A, 357, 219

Persi, P., Marenzi, A. M., Gómez, M., \& Olofsson, G. 2001, A\&A, 376, 907

Persson, S. E., Murphy, D. C., Krzeminski, W., et al. 1998, AJ, 116, 2475

Reipurth, B., \& Bally, J. 2001, ARA\&A, 39, 403 
Shepherd, D. 2003, in In Galactic Star Formation Across the Stellar Mass Spectrum, ed. J. M. De Buizer, \& N. S. van der Bliek (San Francisco: Astronomical Society of the Pacific), ASP Conf. Ser., 287, 333

Shu, F. H., Adams, F. C., \& Lizano, S. 1987, ARA\&A, 25, 237

Shu, F. H., Najita, J. R., Shang, H., \& Li, Z. 2000, in Protostars and Planets IV, ed. V. Mannings, A. Boss, \& S. Russell (Tucson: Univ. Arizona Press), 789

Terebey, S., Shu, F. H., \& Cassen, P. 1984, ApJ, 286, 529

White, R. J., \& Basri, G. 2003, ApJ, 582, 1109
White, R. J., \& Ghez, A. M. 2001, ApJ, 556, 265

Whitney, B. A., \& Hartmann, L. 1992, ApJ, 395, 529

Whitney, B. A., \& Hartmann, L. 1993, ApJ, 402, 605

Whitney, B. A., Kenyon, S. J., \& Gómez, M. 1997, ApJ, 485, 703

Whittet, D. C. B., Prusti, T., Franco, G. A. P., et al. 1997, A\&A, 327, 1194

Wilking, B. A., Greene, T. P., \& Meyer, M. R. 1999, AJ, 117, 469

Wolk, S. J., \& Beck, S. C. 1990, PASP, 102, 745

Zinnecker, H., Krabbe, A., McCaughrean, M. J., et al. 1999, A\&A, 352, L73 